\title{
Wirksam managen im Gesundheitssystem 2010
}

\author{
Die Medizin wandelt sich mit hoher Geschwindigkeit. Erfolgreich werden jene sein, \\ welche die Entwicklungen zu nutzen wissen. College-M vermittelt, wie ein erfolgver- \\ sprechendes, auf die Organisationen des Gesundheitswesens abgestimmtes Manage- \\ ment aussieht. College-M ist der Schweizer Pionier in der Suche nach passenden For- \\ men der Integration von Medizin und Management - integriertes Management.
}

\section{Peter Berchtold, \\ Christof Schmitz \\ Co-Leiter College-M, Bern}

College-M vermittelt im Bereich «Management und Führung im Spital» für die Ärzte sowohl gut verständliche theoretische Grundlagen als auch praxisorientierte Übungsanlagen. Der Unterricht in der Gruppe ist innovativ und kreativ gestaltet, aber dennoch nüchtern fokussiert auf die relevanten Alltagsprobleme. Fazit: eine gute Möglichkeit für Kaderärzte, ihr Rüstzeug in Management und Führung zu erarbeiten und weiterzuentwickeln.

Prof. Dr. med. Thomas J. Neuhaus, Chefarzt Pädiatrie Kinderspital Luzern

Korrespondenz:

PD Dr. Peter Berchtold

College-M

Freiburgstrasse 41

CH-3010 Bern

Tel. 0316323026

Fax 0316323025

weiter.bildung@college-m.ch

www.college-m.ch
Der Nachdiplomkurs «Wirksam Managen» richtet sich an Leitende Ärztinnen und Ärzte, erfahrene Oberärztinnen und -ärzte sowie Führungskräfte aus anderen Berufsgruppen. Angesprochen sind all jene, die Managementwissen sowie praktische Kompetenz für Führungsprozesse erwerben möchten.

Wir vermitteln Managementkompetenz im Rahmen einer lebendigen, interaktiven Lernkultur. Methodisch baut der Nachdiplomkurs auf einem breiten Spektrum verschiedener Lehr- und Vermittlungsformen auf. Der Bogen reicht von Theorie und Evidenzorientierung, Konzepten und Instrumenten des Managens - z. B. der «Leadership Map» - bis zu Selbstreflexionen und zum erfahrungsbasierten Lernen im Rahmen konkreter Projektarbeiten.

Mit dem Nachdiplomkurs können Sie sich

- zielgerichtet in Managementfragen orientieren

- effektiver in den komplexen Netzwerken von Gesundheitseinrichtungen bewegen;

- gesundheitsökonomische und betriebswirtschaftliche Aspekte in die klinische Arbeit mit einbeziehen;

- in Entscheidungssituationen rascher Klarheit gewinnen;

- wirksamer kommunizieren;

- Innovation und Wandel initiieren und Projekte erfolgreich managen.

Der Nachdiplomkurs wird mit einem Zertifikat des College-M abgeschlossen und ist FMH-approved (8 Credits pro Modul).

\section{Aufbau des Nachdiplomkurses}

Element 1: Managen (30.8.-1.9.2010)

- Die Wirksamkeit von Management: Herausforderungen und Antworten

- Zentrale Management- und Führungskonzepte heute

- Systemisches Denken und Handeln als Schlüsselkompetenz im Umgang mit komplexen Situationen

- Leadership Map

- Die Besonderheit von Spitälern und anderen Organisationen im Gesundheitswesen und ihre Konsequenzen fürs Management
Element 2: Führen (27.9.-29.9.2010)

- Teams, Netzwerke, Hierarchien: professionelle Kooperation und Führung

- Führung und Kooperation konkret

- Projekte wirksam managen I

- Einstieg in die Gesundheitsökonomie

Element 3: Intervenieren (10.11.-13.11.2010)

- Gesundheitsökonomische Zusammenhänge und Instrumente - Wirkungen und Nebenwirkungen

- Projekt- und Change Management

Element 4: Wirken (10.1.-12.1.2011)

- Selbstmanagement: Führ' Dich selbst, sonst führt Dich keiner

- Stärkenorientiertes Management

- Kommunikation als Medium von Führung

- Auftrittskompetenz: Wie man Wirkung erzielt

Element 5: Steuern (7.3.-9.3.2011)

- Strategisches Management

- Organisationen entwickeln

- Die Zukunft von Spitälern

Element 6: Integrieren (14.4. - 15.4.2011)

- Die vielfältigen Facetten der Führung integrieren / Leadership Map

- Rückblick und Ausblick

Verantwortliche Programmleiter

- PD Dr. med. Peter Berchtold, Co-Leiter College-M, Bern

- Dr. Christof Schmitz, Co-Leiter College-M, Zürich

Ort: College-M, Freiburgstrasse 41, 3010 Bern

Preis: Fr. 7950.- inklusive aller Programmaktivitäten und Unterlagen

Informationen und Anmeldung: College-M, Freiburgstrasse 41, 3010 Bern, Tel. 03163230 26, Fax 03163230 25, weiter.bildung@college-m.ch, www. college-m.ch 